\title{
IDENTIFICACIÓN DE Porocephalus stilessi (PENTASTOMIDA) EN LA SERPIENTE PERUANA Lachesis muta
}

\author{
Identification of Porocephalus stilessi (Pentastomid) in the Peruvian Snake \\ LACHESIS MUTA
}

\author{
Inés Gárate C. ${ }^{1,3}$, Asucena Naupay I. ${ }^{1}$, Beatriz Suyo L. ${ }^{1}$, Hugo Colquichagua A. ${ }^{1}$, \\ Edith Rodríguez Q. ${ }^{2}$ y ArmandoYarlequé Ch. ${ }^{2}$
}

\section{Resumen}

Se realizó la necropsia a una serpiente venenosa Lachesis muta (Familia Vipiridae), procedente de Satipo, Junín, de $1.95 \mathrm{~cm}$ de longitud y 9 años, que se encontraba cautiva en el serpentario de la Universidad Nacional Mayor de San Marcos, Lima. Se encontró cuatro pentastómidos en la cavidad corporal y en la serosa pulmonar. Los parásitos fueron fijados en formol al 10\% y diafanizados con lactofenol. El estudio morfológico determinó que tres de ellos eran hembras, pues tenían el gonoporo en el extremo posterior y el cuerpo cilíndrico semejante al de un anélido, en tanto que el cuarto parásito era macho, ya que presentaba la parte anterior dilatada y el poro genital en la región anterior, cerca de la boca. La presencia de 4 ganchos, donde los situados en la zona más externa tenían un apéndice poco esclerotizado y frágil, revelaba que eran adultos. Las hembras presentaban 47 anillos y medían $8.3,7.5$ y $6.9 \mathrm{~cm}$ de longitud, y el macho medía $3.7 \mathrm{~cm}$ y tenía 39 anillos. Estas características corresponden a individuos de la especie Porocephalus stilessi, considerándose el primer reporte de esta especie en serpientes del Perú.

Palabras clave: pentastómido, Porocephalus, lachesis, serpiente, parasitismo

\section{Abstract}

A necropsy was conducted in a venomus snake Lachesis muta (Family Vipiridae), originary from Satipo, Junín. This snake was $1.95 \mathrm{~cm}$ long, 9 years old, and was captive at the serpentarium of Universidad Nacional Mayor de San Marcos, Lima. Four pentastomids were found in the corporal cavity and lung serosa. The parasites were fixed in $10 \%$ formol and cleared with lactofenol. The morphologic evaluation revealed that three of them were females (gonopore close to the end body and cylindrical body shape similar to an annelid) and one male (expanded front body and genital pore near the mouth). The parasites had

\footnotetext{
${ }^{1}$ Laboratorio de Parasitología Humana y Animal, ${ }^{2}$ Laboratorio de Biología Molecular, Facultad de Ciencias Biológicas, Universidad Nacional Mayor de San Marcos, Lima

${ }^{3}$ E-mail:igarateca@yahoo.com
} 


\begin{abstract}
4 hooks where two of them showed some sclerotic appendixes compatible with adult forms. Females had 47 rings and a length of $8.3,7.5$ and $6.9 \mathrm{~cm}$, respectively, while the male had 39 rings and a length of $3.7 \mathrm{~cm}$. These characteristics corresponded to the species Porocephalus stilessi. This is the first report of this parasite in Peruvian snakes.
\end{abstract}

Key words: pentastomids, Porocephalus, lachesis, snake, parasitism

\section{INTRODUCCIÓN}

Los pentastómidos son parásitos del sistema respiratorio de vertebrados. Los estadios adultos se encuentran mayormente en los pulmones de reptiles, especialmente en serpientes, lagartijas y cocodrilos. Una especie vive en los sacos aéreos de gaviotas y golondrinas marinas y otra habita en la nasofaringe de cánidos y félidos y, ocasionalmente, como ninfa en la nasofaringe de seres humanos. Así pues, los pentastómidos tienen interés desde el punto de vista zoológico $\mathrm{y}$, eventualmente, importancia médica (Schmidt y Roberts, 1984).

Según Self (1969), el phylum Pentastomida incluye dos órdenes (Fain, 1966): Cephalobaenida y Porocephalida. En ésta última se encuentra la Familia Porocephalidae donde se ubican los pentastómidos de las vías respiratorias de ofidios. Uno de los géneros de esta familia es el Porocephalus, parásito de ofidios africanos y sudamericanos con las especies $P$. crotali, $P$. clavatus, $P$. stilessi y $P$. subuliffer.

Los adultos de $P$. crotali son comunes en vipéridos del género Bothrops, mientras que los adultos de $P$. clavatus se encuentran en boas. Hasta la fecha, se considera que $P$. stilessi (Sambon, 1910) es específico para las serpientes del género Lachesis.

El ciclo biológico de estos pentastómidos es muy complejo. Las hembras adultas que habitan dentro del pulmón de los ofidios, eliminan periódicamente huevos larvados, que son deglutidos y eliminados con la materia fecal. Una vez ingeridos los huevos por el hospedero intermediario (roedores, herbívoros, carnívoros, primates e incluso al hombre), eclosionan en el tubo digestivo y las larvas perforan la pared intestinal donde se enquistan o llegan por vía sanguínea a otros órganos (hígado, riñón, pulmones, ganglios, cavidad visceral) donde también se pueden enquistar. Tras varias mudas se transforman en ninfas. El huésped definitivo se infecta ingiriendo las ninfas enquistadas, las cuales penetran la pared intestinal y llegan a los pulmones donde se desarrollan hasta adultos sexualmente maduros, repitiendo el ciclo. Durante el pasaje de las larvas y ninfas hasta su localización definitiva, se producen lesiones que provocan granulomas en distintos órganos y lesiones inflamatorias en los pulmones, aunque en ciertos casos no se producen reacciones (Cheng, 1986).

Lachesis muta, conocida como "shushupe" o "bushmaster", es la serpiente sudamericana de mayor tamaño pues alcanza a medir desde 1.8 hasta $4 \mathrm{~m}$ de longitud. Se trata de una serpiente solenoglifa; es decir, posee dos colmillos retráctiles y acanalados por los cuales vierte el veneno, cuyo peso seco puede variar entre 1.5 y 2 g. Es una serpiente terrestre que vive en la floresta y aunque posee capacidad para trepar los árboles, prefiere alojarse en lugares cercanos al suelo. Sus presas son roedores grandes tales como ronsocos, comadrejas, ardillas, ratas silvestres y, eventualmente, puede comer cerdos de mediano peso. Aunque no ataca al hombre, si llegara a morderlo le va a producir efectos locales muy agudos como dolor intenso y hemorragia; así mismo, los 
efectos sistémicos como la hipotensión y coagulación sanguínea pueden causar la muerte.

En la preparación de los antivenenos se requiere contar con el veneno de la serpiente. La L. muta puede ser mantenida en serpentarios, aunque muestra poca tolerancia al cautiverio a diferencia de sus congéneres como Bothrops atrox. Los reportes sobre el tema señalan una supervivencia promedio no mayor a seis meses, por lo que se requiere de estudios destinados a explorar las causas de esta limitante (Yarlequé, 2000).

\section{Materiales y Métodos}

A un ejemplar hembra de la serpiente Lachesis muta, de $1.95 \mathrm{~m}$ de longitud y con edad aproximada de 9 años, proveniente de Satipo, Junín, que estuvo en cautiverio en el serpentario del Museo de Historia Natural

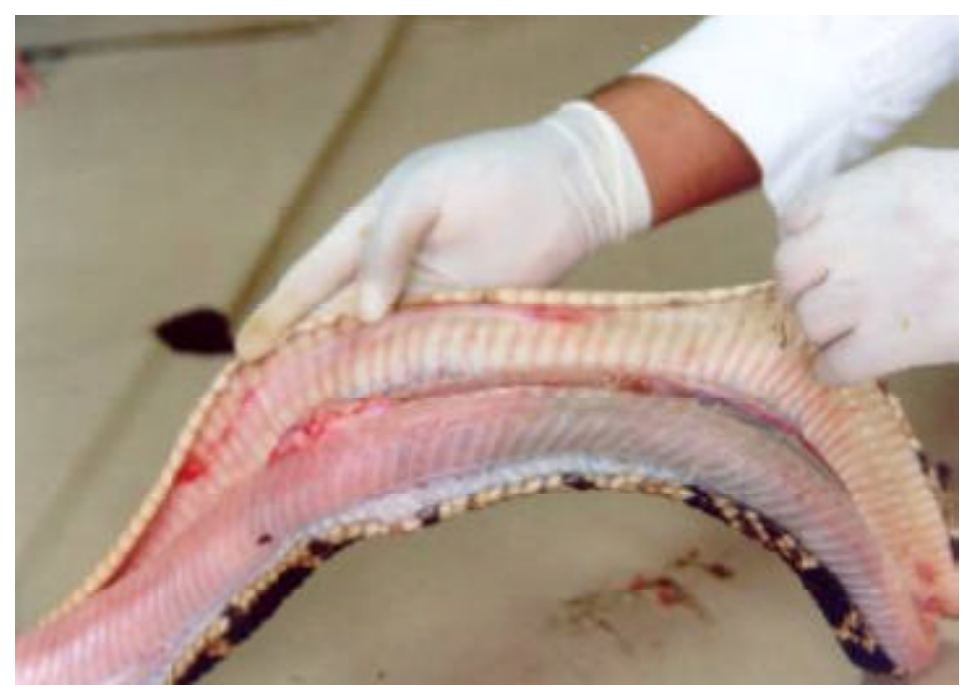

Figura 1. Necropsia y extracción de cuatro pentastómidos de la cavidad corporal y serosa pulmonar de Lachesis muta.

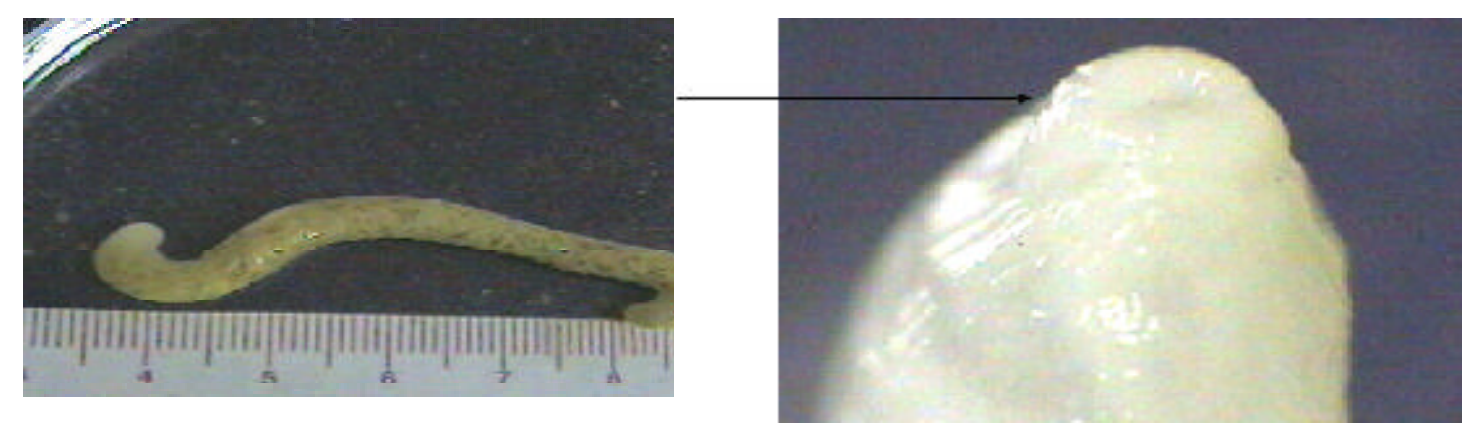

Figura 2. Pentastómido de la especie Porocephalus stilessi (a) Especimen hembra. (b) Gonoporo 

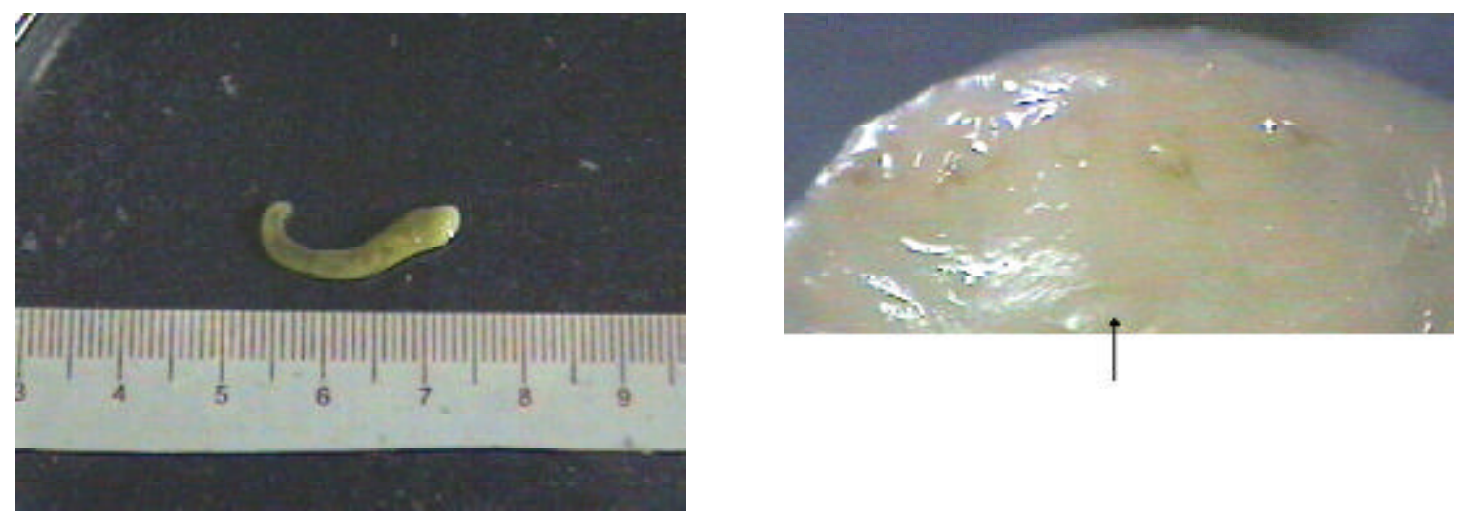

Figura 3. Pentastómido de la especie Porocephalus stilessi (a) Especimen macho (b) Parte anterior y poro genital

de la Universidad Nacional Mayor de San Marcos, Lima, se le hizo una necropsia seis horas después de su muerte natural. Se encontró un parásito en la cavidad corporal y otros tres en la serosa pulmonar (Fig. 1), que fueron fijados en formol comercial al $10 \%$.

\section{Resultados}

En la observación microscópica se pudo constatar que tres ejemplares eran hembras pues presentaban el cuerpo cilíndrico, semejante al de un anélido, y el gonoporo en el extremo posterior (Fig. 2); mientras que el cuarto ejemplar era macho, pues presentaba la parte anterior dilatada y el poro genital en la región anterior, cerca de la boca (Fig. 3). La presencia, en todos los individuos, de 4 ganchos, donde los situados en la zona más externa tenían un apéndice poco esclerotizado y frágil, revelaba que eran ejemplares adultos (Fig. 6). Las hembras presentaban 47 anillos y medían $8.3,7.5$ y $6.9 \mathrm{~cm}$ de longitud, respectivamente; el macho medía $3.7 \mathrm{~cm}$ y tenía 39 anillos. Las características señaladas corresponden a $P$. stilessi, considerándose como el primer reporte de esta especie en serpientes del Perú. Estos resultados concuerdan con lo señalado por Riley y Self (2004), respecto a que $P$. stilessi es específico de las serpientes del género Lachesis.

\section{Discusión}

Los pentasósmidos ocasionan perjuicio al hospedador, y en algunos casos pueden causar su muerte, sobre todo si son sometidos a estrés producido por cautiverio. Tantaleán y Gozalo (1985) identificaron por primera vez a $P$. crotali en pulmones de Bothrops atrox, señalando que les causan daños con sus garfios y por sus hábitos hematófagos. De manera similar, se puede asumir que los pentastómidos encontrados en el presente estudio, habrían ocasionado daños severos en la serosa pulmonar de la serpiente L. muta, generando procesos inflamatorios, hemorragia y anemia.

Aguiar et al. (1999), revisando 12 especímenes de L. muta provenientes de la floresta de Brasil, encontraron que la mitad estaba parasitada por estos pentastómidos, precisando que su presencia es perjudicial para sus hospedadores.

\section{Literatura Citada}

1. Aguiar AS, Rubião ECN, Bastos OMP, Melgarejo AR. 1999. Parasitismo por Porocephalus stilessi (Sambon, 1910) (Pentastomida) en Lachesis muta rhombeata Wied, 1824 (Serpentes: 
Viperidae). En: V Congreso Latinoamericano de Herpetología. Montevideo.

2. Cheng TC. 1986. General parasitology. Orlando: Academic Press, College Division. $2^{\text {nd }}$ ed. $\mathrm{p}$ 765-773.

3. Fain A. 1966. Pentastomida of snake. Their parasitological role in man and animals. Mem Inst Butantan 33(1): 167174.

5. Riley J, Self JT. 1979. On the systematics of the pentastomid genus Porocephalus (Humboldt, 1811) with descriptions of two new species. Syst Parasitol 1(1): 25-42.
6. Schmidt GD, Roberts L. 1984. Fundamentos de parasitología. México: Ed. Continental. $680 \mathrm{p}$.

7. Self J. 1969. Biological relationships of the Pentastomida: A bibliography on the Pentastomida. Exp Parasitol 24: 63-119.

8. Tantaleán M, Gozalo A. 1985. Parásitos de Bothrops atrox (Viperidae) de la Amazonía Peruana. Rev Asoc Med Vet Esp Anim Peq (Perú) 20: 11-12.

9. Yarlequé A. 2000. Las serpientes peruanas y sus venenos. Lima: Fondo Editorial UNMSM. 78 p. 